\title{
The effect of sustained, long-term changes in alcohol intake on cardiovascular risk
}

\author{
Ulla Toft ${ }^{1 *}$, Charlotta Pisinger ${ }^{1}$, Mette Aadahl ${ }^{1}$, Allan Linneberg ${ }^{1}$, Cathrine Lau ${ }^{2}$, \\ Torben Jørgensen ${ }^{1}$ \\ ${ }^{1}$ Research Centre for Prevention and Health, Glostrup, Denmark; ${ }^{*}$ Corresponding Author: ulla.toft $@$,regionh.dk \\ ${ }^{2}$ Steno Diabetes Center, Gentofte, Denmark
}

Received 11 August 2012; revised 21 September 2012; accepted 30 October 2012

\begin{abstract}
Objective: To investigate whether sustained longterm changes in alcohol intake are predictive of cardiovascular risk. Methods: The study population was a subpopulation of the five-year intervention study, Inter99 study, (1999-2006), Copenhagen, Denmark ( $n=2117 ; 30$ - 60 years). Alcohol intake was assessed by questionnaires at baseline, one-, three- and five-year follow-up. The associations between sustained long-term changes in alcohol intake and cardiovascular risk factors (HDL and non-HDL cholesterol, systolic and diastolic blood pressure (BP); the absolute risk of ischemic heart disease (CRS)) at five-year follow-up were explored by linear regression models. The alcohol variables were tested for linear association with the response variable. Results: Sustained increased alcohol intake was significantly associated with increased CRS ( $\beta=0.0028 ; P=0.006)$ and a decreased HDL cholesterol $(\beta=-0.0028 ; P=0.005)$. Among participants with a moderate overall alcohol intake at baseline increased alcohol intake was significantly associated with an increased plasma triglyceride $(\beta=0.0069 ; P=0.04)$. No association with triglyceride was found for participants with a high alcohol intake. Change in wine intake was significantly negatively associated with changes in diastolic $B P(\beta=0.0015 ; P=$ 0.02). Conclusions: Sustained increase in the long-term intake of alcohol was a significant risk factor for an increased CRS, increased triglyceride level and decreased HDL cholesterol. Increased wine intake was associated with decreased diastolic BP.
\end{abstract}

Keywords: Alcohol Consumption; Cardiovascular Disease; Intervention Studies

\section{INTRODUCTION}

Health aspects of alcohol have been debated for centuries. There are both beneficial and harmful implications of alcohol in cardiovascular disease. Benefits of a moderate alcohol intake may be overstated in many observational studies due to inclusion of abstainers who had quit due to poor health [1] and inadequate adjustments for important confounders [2-4]. In controlled trials alcohol intake has been found to have favourable short term (weeks) effects on for example high-density lipoprotein (HDL) levels, which suggest at least some cardiometabolic benefit of moderate alcohol intake. However, the long-term effects are unclear and few studies have investigated the effect of a sustained change in alcohol intake. Furthermore, possible beneficial effects on some cardiovascular risk factors may be counteracted by adverse effects on other risk factors. It therefore seems relevant to investigate the effect of changes in alcohol intake on a measure of the overall risk of ischemic heart disease (IHD).

The aim of the present study was to investigate whether sustained long term change in alcohol intake is predictive of both single cardiovascular risk factors and the absolute risk of IHD.

\section{METHODS}

\subsection{Study Population}

The study population consisted of a subgroup of the participants in the Inter99 study, which is described in details earlier [5].

Briefly, the Inter99 study was a large population-based intervention study including individualised risk assessment; multi-factorial, non-pharmacological intervention on lifestyle; and a program for maintenance. The study group was all individuals aged $30-60$ years living in 11 municipalities in the suburbs south-west of Copenhagen City $(\mathrm{N}=61,301)$. An age- and sex-stratified random sample of 13016 individuals was drawn from the Civil 
Registration and invited for the five-year intervention study (high intensity group: $\mathrm{N}=11.708$; low intensity group: $\mathrm{N}=1308)$. A total of $6784(52.5 \%)$ accepted and were included in the intervention. The Inter99 study was approved by the local Ethics Committee (KA 98 155) and is registered with Clinical Trials gov. (NCT00289237).

In this study participants were only included in the analyses if they participated during the entire five-year study period and if they answered the questions on alcohol intake $(n=3536)$. Because the aim was to investigate the effect of a sustained long-term (three years) change in alcohol intake, participants that changed their usual alcohol intake substantial (defined as \pm 3 drinks per week) in the maintenance period (from three- to five-year follow-up) were excluded ( $\mathrm{n}=1128)$. Furthermore abstainers at baseline, three or five-year follow-up $(n=159)$ and participants in medical treatment for hypertension or hypercholesterolemia $(\mathrm{n}=132)$ were excluded, leaving 2117 for the analyses.

\subsection{Physical Examinations and Risk Assessment}

All participants in the intervention group were required to fast from midnight on the day before attendance and all had a thorough health examination. For each participant the absolute total risk of IHD within the next 10 years (the Copenhagen Risk Score, CRS) was estimated using the computer programme PRECARD ${ }^{\circledR}$ [6]. Individuals were categorized as high-risk individuals if they had either an absolute risk in the upper quintile of the distribution, stratified according to sex and age or at least one of the following isolated risk factors: systolic blood pressure $(\mathrm{BP}) \geq 160 \mathrm{mmHg}$ and/or in antihypertensive treatment, total cholesterol $\geq 7.5 \mathrm{mmol} / \mathrm{l}$ and/or treated with statins, body mass index (BMI) $\geq 30 \mathrm{~kg} / \mathrm{m}^{2}$, glucose intolerance or diabetes [7], or were daily smokers.

\subsection{Intervention}

Each participant had a lifestyle consultation focussing on smoking, physical activity, diet and alcohol. Participants were encouraged to keep their alcohol intake below the official recommended limits $(21 / 14$ drinks per week for men/women).

In addition to the individual counselling, high risk individuals in the high intensity group were offered group counselling on diet and exercise or smoking cessation/ reduction. The participants were taught that alcohol may increase their risk of weight gain and hypertension (For more details: www.inter99.dk ).

\subsection{Follow-Up}

High-risk participants in the intervention group were re-invited after one and three years for a health examina- tion, completion of questionnaires, risk assessment and individual lifestyle counselling. Individuals who were still at high risk of IHD were again offered group counselling. Low-risk participants were followed by questionnaires. At five-year follow-up all participants from baseline were invited for health examination and a short lifestyle consultation.

\subsection{Assessment of Dependent Variables}

At the health examinations at baseline and five-year follow-up, participants provided fasting blood samples. Total cholesterol, HDL and triglyceride were measured by enzymatic procedures (Boeringer Mannhein, Germany). Non-HDL was calculated by subtracting HDL cholesterol from total cholesterol.

BP was measured twice with a mercury sphygmomanometer after $5 \mathrm{~min}$. of rest in a lying position. Height was measured without shoes to the nearest $0.5 \mathrm{~cm}$ and weight was measured without shoes and overcoat to the nearest $0.1 \mathrm{~kg}$. Body mass index (BMI) was calculated $\left(\mathrm{kg} / \mathrm{m}^{2}\right)$. Waist circumference was measured midway between the lower rib margin and iliac crest.

The 10-year absolute risk of IHD were estimated by the CRS which is calculated based on sex, age, heredity, former IHD, diabetes, height, weight, smoking habits, total serum cholesterol, serum HDL cholesterol and BP [8]. Information on lifestyle and intake of medication was obtained by a questionnaire.

\subsection{Alcohol Intake}

Alcohol intake was assessed by questionnaires both at baseline, three and five year follow-up. The participants were asked, on a continuous scale, to report their average weekly intakes of regular beer, strong alcoholic beer, wine, dessert wine, and spirits/strong drink. The reported intakes of alcoholic beverages was calculated into units of ethanol ( 1 unit $=1.5 \mathrm{cl} / 12 \mathrm{~g}$ ) and summarized to total amount of drinks per week. High intake of alcohol was defined as an intake above the recommended (in Denmark at the time of the intervention) maximum level of 14/21 drinks per week for women and men, respectively. Changes in alcohol intake from baseline to three-year follow-up were calculated by subtracting the reported baseline intake from the reported intake at three-year follow-up. To assess if the changes were maintained, the alcohol intake at three-year follow-up were subtracted from the reported intake at five-year follow-up. Sustained changes were defined as a change of not more that \pm 3 drinks per week in the maintenance period (from three- to five-year follow-up).

\subsection{Covariates}

Age and sex were known from the Danish Civil Reg- 
istration System. Education, physical activity, smoking and dietary intake were assessed by questionnaires. Education was defined on the basis of questions regarding number of years of vocational training categorized into four classes: 1 : none, $2: \leq 1$ year, $3: 2-4$ years, and $4:>4$ years. Physical activity was measured as number of minutes being physical active during transport to and from work and/or at leisure time per week. Changes were calculated as the difference between the measure at baseline and five year follow-up.

Dietary habits were measured using a validated dietary quality score, The Dietary Quality Score, developed from a 48-item food frequency questionnaire. The development and validation of the Dietary Quality Score has been described earlier [9]. Changes in dietary habits were calculated by subtracting the participants score at baseline from the score at five-year follow-up.

Smoking habits was recorded in five categories: daily smokers, occasional smokers ( $<1 \mathrm{~g}$ of tobacco per day); ex-smokers; stopped smoking after the start of the Inter99 study; never smokers.

\subsection{Statistical Analysis}

Data were analysed using SAS statistical software, version 9.2 (SAS Institute Inc., Cary, NC, USA). Changes in alcohol from baseline to three year follow-up were included as the explanatory variable in the statisticcal analyses. The associations between three-year changes in alcohol intake and cardiovascular risk factors at fiveyear follow-up were explored by a series of linear regression models with the five-year follow-up values of each cardiovascular risk factor as outcome variable and adjusted for the baseline level of each risk factor. The interpretation of the results is then the effect on changes in the risk factor from baseline to five-year follow-up. The alcohol variables were thoroughly tested for linear association with the response variable, testing for a cubic, quadratic or a linear spline association.

Cardiovascular risk factors investigated were: HDL, non-HDL, systolic and diastolic BP, triglyceride, and CRS. Separate analyses for total alcohol intake (without considering beverage type) and the percentage of alcohol intake consumed as wine, beer or spirits/strong drinks were performed. Adjustment for sex, age, self-reported smoking status at baseline and five-year follow-up, changes in dietary intake and physical activity were made. All analyses were carried out with and without adjustment for weight change from baseline to five-year follow-up.

Outcome variables were logarithm transformed if not normally distributed and residual plots were made of each model to check if the residuals were randomly dispersed around the horizontal axis. Interactions between the change in alcohol intake and sex, age or high/mod- erate alcohol intake at baseline were explored by including the interaction terms in the models. In case of significant interaction, the interaction term was included in the model and separate parameter estimates were calculated. A P-value of $5 \%$ or less was considered significant.

\section{RESULTS}

Baseline characteristics are presented in Table 1. The model control analyses showed that the outcome variables BP, triglyceride and CRS were not normally distributed. Logarithm transformation of these variables improved the residual plots markedly and therefore we used natural logs of BP, triglyceride level and CRS in all analyses including these variables.

Significant interactions were found between level of

Table 1. Baseline characteristics and alcohol intake according to sex in the 2117 subjects included in this study.

\begin{tabular}{|c|c|c|}
\hline & $\begin{array}{c}\text { Men (\%) } \\
\mathrm{n}=982\end{array}$ & $\begin{array}{c}\text { Women }(\%) \\
\mathrm{n}=1135\end{array}$ \\
\hline \multicolumn{3}{|l|}{ Age (years) } \\
\hline $30-35$ & 15 & 14 \\
\hline $40-50$ & 62 & 65 \\
\hline $55-60$ & 22 & 21 \\
\hline \multicolumn{3}{|l|}{ Vocational training } \\
\hline None & 11 & 13 \\
\hline$<2$ years & 2 & 6 \\
\hline $2 \leq$ years $\leq 4$ & 68 & 72 \\
\hline$<4$ years $\leq 9$ & 19 & 9 \\
\hline \multicolumn{3}{|l|}{ Daily smoking } \\
\hline Yes & 26 & 22 \\
\hline \multicolumn{3}{|l|}{ Dietary habits } \\
\hline Healthy & 16 & 31 \\
\hline Average & 73 & 65 \\
\hline Unhealthy & 11 & 4 \\
\hline \multicolumn{3}{|l|}{$\begin{array}{l}\text { Leisure time physical } \\
\text { activity }\end{array}$} \\
\hline Mainly sedentary & 17 & 14 \\
\hline Moderate activity & 58 & 71 \\
\hline Regular/heavy exercise & 25 & 15 \\
\hline \multicolumn{3}{|l|}{$\begin{array}{l}\text { Binge drinking } \\
\text { (days the last week) }\end{array}$} \\
\hline None & 59 & 71 \\
\hline 1 & 26 & 20 \\
\hline $2-3$ & 12 & 8 \\
\hline \multirow[t]{2}{*}{$4-7$} & 3 & 1 \\
\hline & \multicolumn{2}{|c|}{ Median (P5, P95) } \\
\hline Wine $^{a}$ & $3(0-15)$ & $3(0-15)$ \\
\hline Regular beer $^{\mathrm{a}}$ & $2(0-15)$ & $1(0-6)$ \\
\hline Strong alcoholic beer ${ }^{\mathrm{a}}$ & $0(0-3)$ & $0(0-3)$ \\
\hline Spirits/strong drinks ${ }^{\mathrm{a}}$ & $0(0-4)$ & $0(0-2)$ \\
\hline Dessert wine $^{a}$ & $0(0-1)$ & $0(0-1)$ \\
\hline Total alcohol intake & $8(2-29)$ & $5(1-19)$ \\
\hline
\end{tabular}

${ }^{\text {a }}$ Drinks per week. Abbreviations: ${ }^{b} \mathrm{P} 5=5^{\text {th }}$ percentile; $\mathrm{P} 95=95^{\text {th }}$ percentile. 
alcohol intake at baseline (high/moderate) and both beer intake (with HDL as the dependent variable) and overall alcohol intake (with triglyceride as the dependent variable). No other significant interactions were found with level of alcohol intake and no significant interactions were found with age or sex.

Increased intake of alcohol overall during three years of intervention in the Inter99 study was significantly associated with an increased absolute risk of IHD according to the CRS $\left(\beta=0.0028 ; \mathrm{P}=0.006 ; \mathrm{R}^{2}=0.870\right.$; Figure 1) and a decreased HDL cholesterol level $(\beta=$ $-0.0028 ; \mathrm{P}=0.005 ; \mathrm{R}^{2}=0.709$; Figure 2) at five-year follow-up. Among participants with a moderate overall alcohol intake at baseline an increased intake of alcohol at three year follow-up was significantly associated with a increased plasma triglyceride level at five-year follow-up $\left(\beta=0.0069 ; \mathrm{P}=0.05 ; \mathrm{R}^{2}=0.463\right.$; Figure 3$)$. No association with triglyceride level was found for participants with a high alcohol intake. A borderline significant association was found between changes in alcohol intake and diastolic $\mathrm{BP}(\beta=0.0008 ; \mathrm{P}=0.053$, data not shown $)$. No significant associations were found between changes in overall alcohol intake and changes in systolic BP and non-HDL cholesterol.

Similar to overall alcohol intake, an increased intake of beer was significantly associated with increased CRS $\left(\beta=0.0046 ; \mathrm{P}=0.03 ; \mathrm{R}^{2}=0.875 ;\right.$ data not shown $)$ at five-year follow-up. Increased intake of beer was furthermore associated with decreased HDL level among participants with a high alcohol intake $(-0.0086 ; \mathrm{P}=$ $0.003)$, whereas a positive association was found between beer intake and HDL level among participants with a moderate alcohol intake at baseline $(\beta=0.012$; $\mathrm{P}$ $=0.04 ; \mathrm{R}^{2}=0.688$; data not shown). No associations were found between changes in beer intake and changes in BP, triglyceride and non-HDL cholesterol.

Three year change in wine intake was significantly negatively associated with changes in the diastolic BP at five-year follow-up $\left(\beta=-0.0015 ; \mathrm{P}=0.02 ; \mathrm{R}^{2}=0.347\right.$; Figure 4).

No associations were found between the intakes of spirit/strong drink and the different risk factors. Furthermore no significant associations were found when the intake was expressed as the percentage of alcohol consumed as wine, beer or spirit/strong drink. Adjustments for weight change did not markedly change in the associations investigated and did not change any of the conclusions made above.

\section{DISCUSSION}

According to the results of this study total alcohol in-

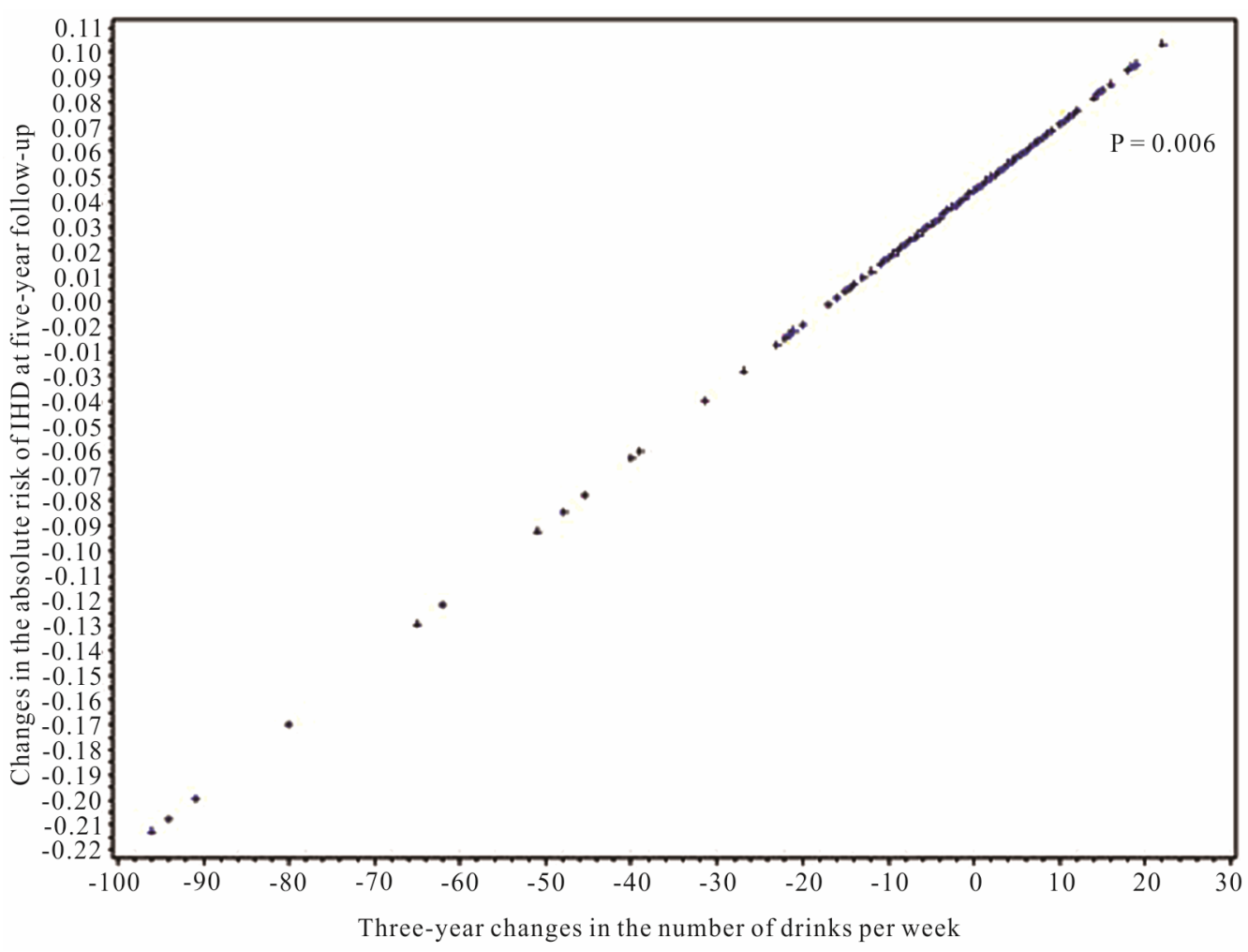

Figure 1. The regression line for the association between three-year changes in alcohol intake and the absolute risk of IHD at five-year follow-up (Results are adjusted for diastolic BP at baseline, age, sex, education and five year changes in smoking, physical activity and diet). 


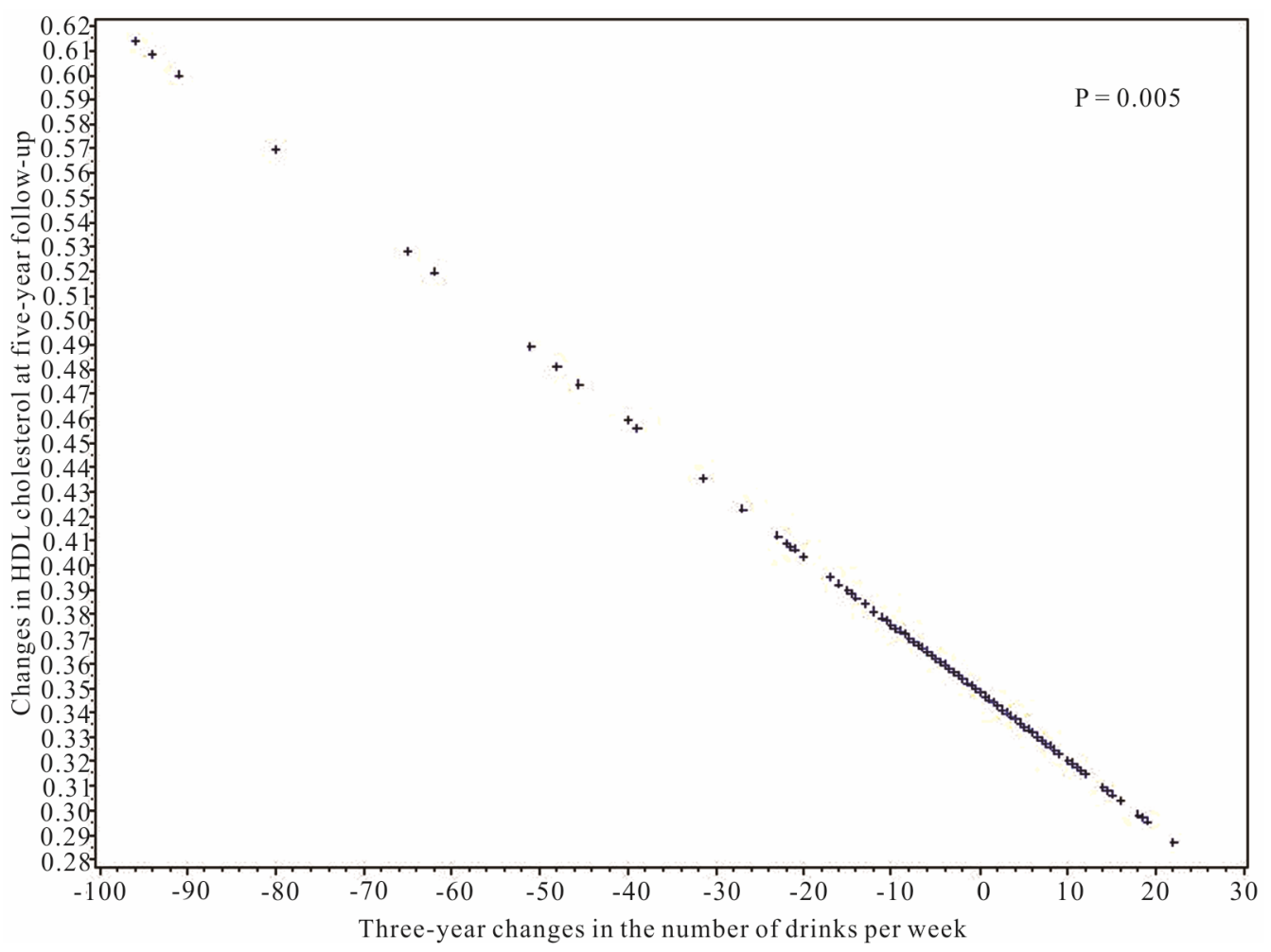

Figure 2. The regression line for the association between three-year changes in alcohol intake and HDL cholesterol at five-year follow-up (Results are adjusted for diastolic BP at baseline, age, sex, education and five year changes in smoking, physical activity and diet).

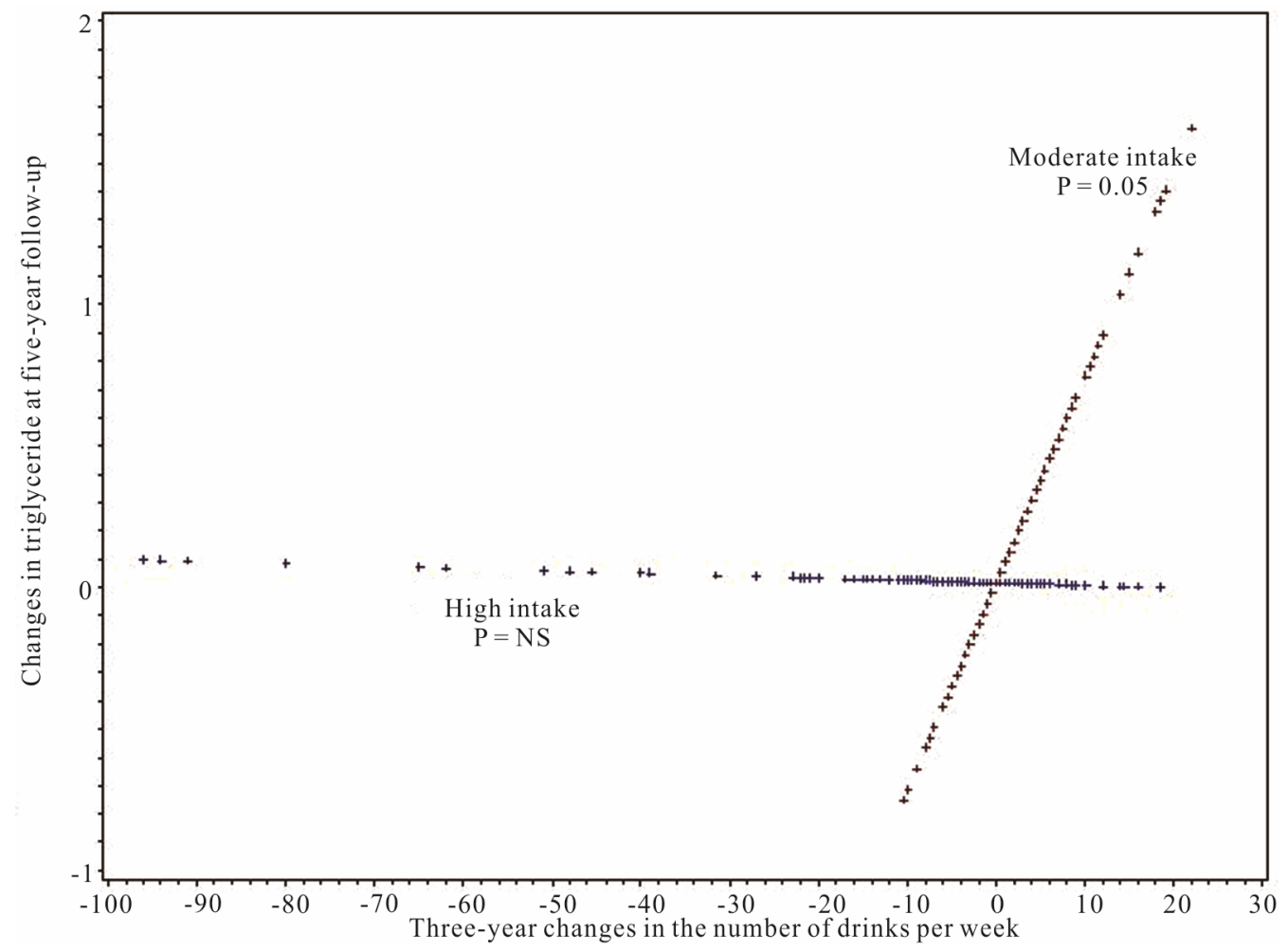

Figure 3. The regression line for the association between three-year changes in alcohol intake and triglyceride levels at five-year follow-up (Results are adjusted for diastolic BP at baseline, age, sex, education and five year changes in smoking, physical activity and diet). 


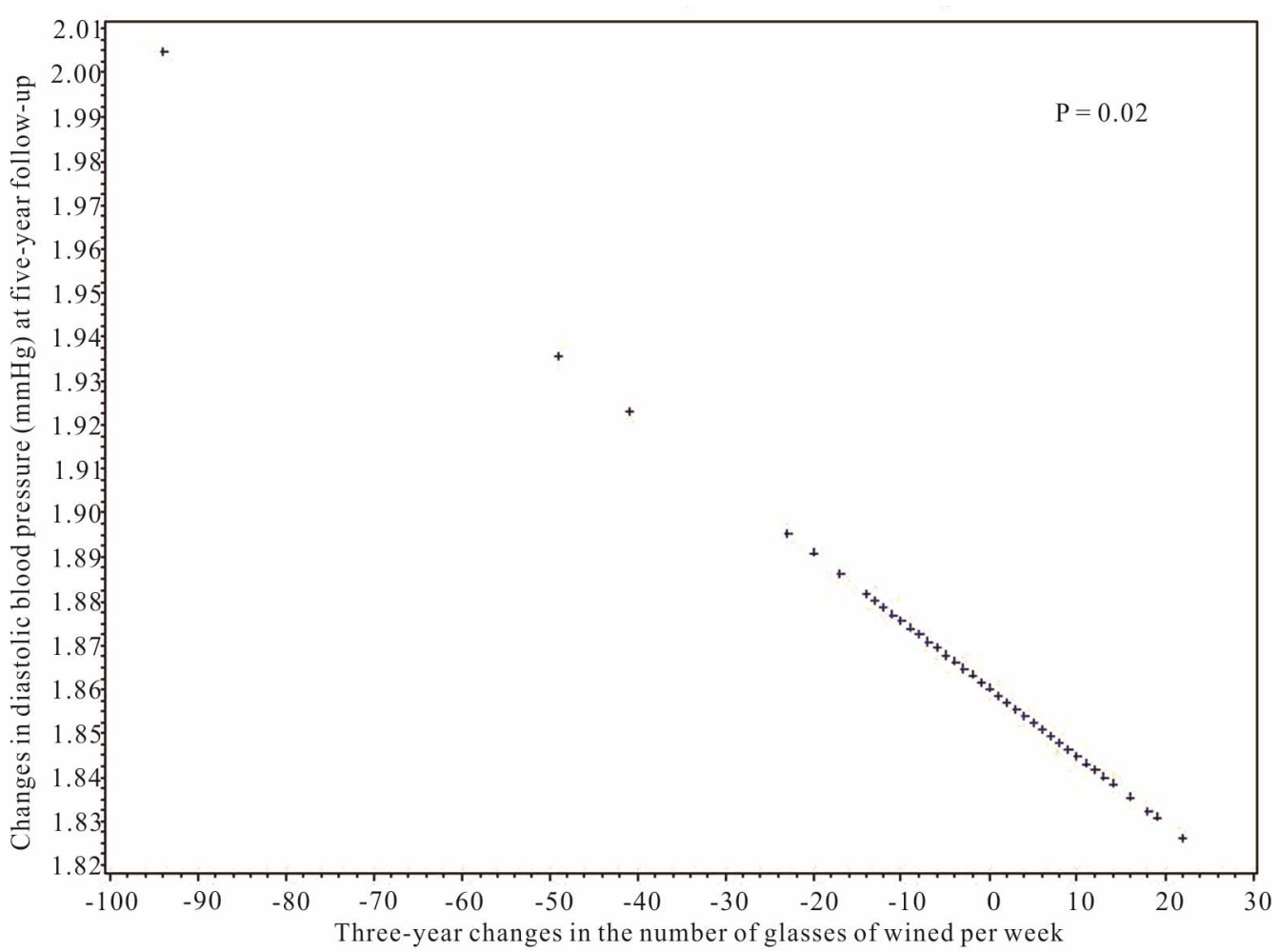

Figure 4. The regression line for the association between three-year changes in wine intake and the diastolic blood pressure at five-year follow-up (Results are adjusted for diastolic BP at baseline, age, sex, education and five year changes in smoking, physical activity and diet).

take is a significant risk factor for increased triglyceride level and increased absolute risk of IHD. Surprisingly, decreased overall alcohol intake and beer intake (only among participants with a high alcohol intake at baseline) was associated with increased HDL cholesterol level. Furthermore, increased intake of wine (and overall alcohol intake, borderline) was associated with decreased diastolic BP.

The present study contradicts with the conclusions of many (but not all) earlier observational studies that have shown a J-shaped relation between intake of alcohol and the risk of heart disease, and thus a protective effect of a moderate alcohol intake [10]. Nevertheless the beneficial health effects of alcohol are discussed and increasing evidence indicates serious methodological problems in many of the observational studies.

A major methodological problem in many studies is the inclusion of abstainers in the analyses. Abstainers have repeatedly been found to be significantly more likely to have a history of alcoholism, be older, have lower SEP, more co-morbidity, worse mental health, more unhealthy lifestyle, and worse health in general than individuals with a small alcohol intake $[2,3,11]$. And becoming an abstainer is a phenomenon found to be associated with aging or bad health [2]. A meta-analyses of 54 studies identified studies that properly took this into account and these studies did not find any protective health effect of alcohol [1]. Therefore, in this study we excluded abstainers both at baseline and follow-up.

Another problem in earlier studies has been that alcohol intake in many questionnaires has been assessed by categorical questions. This makes it difficult to assess the intake with the lowest risk. An earlier Danish study took this into account by using generalized additive models and by excluded abstainers and found a linear increased mortality with increasing alcohol intake and concluded that the J-shaped curve found in most studies is caused by a higher risk in abstainers rather than a health benefit of alcohol [12]. The alcohol intake in this study was assessed on a continuous scale.

Finally, Corrao et al. [13] found evidence for publication bias for the effect of moderate intake of alcohol. Similar results were recently found by Kodama et al. [14]. However, this issue is also still discussed [15].

In this study we found that long term increases in alcohol intake was associated with an increased triglyceride level among individuals with a moderate alcohol intake. This finding is in accordance with the finding of many other earlier studies [16-18].

In controlled settings alcohol has repeatedly been found to cause short-term increases in HDL cholesterol [19-21]. Surprisingly, in this study we found an inverse associa- 
tion between long-term and sustained changed in alcohol intake and the changes in HDL cholesterol from baseline to five-year follow-up. This result could indicate that the body may adapt to a new level of alcohol intake and that the acute positive effect might diminish in the long-term.

A significant inverse association between long-term changes in wine intake and changes in diastolic BP was found. This finding is in accordance with the finding for wine, in some earlier observational studies $[22,23]$ but not all [24]. The high potassium content in wine might be protective against hypertension and thereby explain the possible beneficial effect of wine.

Important strengths of this study are is the prospective design which provides the opportunity to investigate the effect of changes in alcohol intake and thus the indication of a causal relationship between alcohol intake and health becomes stronger than the conclusions from cross sectional studies. Other strengths are the repeated measures of both alcohol and several cardiovascular risk factors; that the study was performed in a general population, the thorough adjustments for confounders; and that we looked at the absolute risk of IHD rather than just the effect on single risk factors.

A noteworthy limitation of the present study is that we were not able to include the non-treatment control group in the Inter99 study as risk factor measurements were not available for this group. Additionally, the alcohol intake was self-reported and we only looked at the association with different biomarkers which are intermediary factors on the pathway between lifestyle and development of chronic disease.

In conclusion sustained increase in the long term overall intake of alcohol was found to be a significant risk factor for the absolute risk of IHD, increased triglyceride level and decreased HDL cholesterol level. Increased wine intake was associated with decreased diastolic BP.

\section{ACKNOWLEDGEMENTS}

We acknowledge all members of the Inter99 team and the steering committee of the Inter99 study: Torben Jørgensen, DMSc (PI), Knut Borch-Johnsen, DMSc (Co-PI), Hans Ibsen, DMSc, Troels Thomsen, Ph.D, Charlotta Pisinger, $\mathrm{PhD}$ and Charlotte Glümer, $\mathrm{PhD}$.

The study was initiated and analyzed by the investigators in this project. The work was supported economically by: The Danish Medical Research Council, The Danish Centre for Evaluation and Health Technology Assessment, Novo Nordisk, Copenhagen County, The Danish Heart Foundation, The Danish Pharmaceutical Association, Augustinus foundation, Ib Henriksen foundation and Becket foundation.

\section{REFERENCES}

[1] Fillmore, K.M., Stockwell, T., Chikritzhs, T., Bostrom, A. and Kerr, W. (2007) Moderate alcohol use and reduced mortality risk: Systematic error in prospective studies and new hypotheses. Annals of Epidemiology, 17, S16-S23. doi:10.1016/j.annepidem.2007.01.005

[2] Naimi, T.S., Brown, D.W., Brewer, R.D., et al. (2005) Cardiovascular risk factors and confounders among nondrinking and moderate-drinking US adults. American Journal of Preventive Medicine, 28, 369-373. doi:10.1016/j.amepre.2005.01.011

[3] Zarkin, G.A., French, M.T., Mroz, T. and Bray, J.W. (1998) Alcohol use and wages: New results from the national household survey on drug abuse. Journal of Health Economics, 17, 53-68. doi:10.1016/S0167-6296(97)00023-4

[4] Tjonneland, A., Gronbaek, M., Stripp, C. and Overvad, K. (1999) Wine intake and diet in a random sample of 48763 Danish men and women. American Journal of Clinical Nutrition, 69, 49-54.

[5] Jorgensen, T., Borch-Johnsen, K., Thomsen, T.F., Ibsen, H., Glumer, C. and Pisinger, C. (2003) A randomized nonpharmacological intervention study for prevention of ischaemic heart disease: Baseline results Inter99 (1). European Journal of Preventive Cardiology, 10, 377-386. doi:10.1097/01.hjr.0000096541.30533.82

[6] Thomsen, T., Borch-Johnsen, K., Davidsen, M. and Ibsen, H. (1997) The "PRECARD" study: Identificattion and management of individuals at risk of developing cardiovascular disease. Canadian Journal of Cardiology, 13, 286B-287B.

[7] World Health Organisation (1999) Definition, diagnosis and classification of diabetes mellitus and its complications. Report of a WHO consulation, part 1: Diagnosis and classification of diabetes mellitus, Geneva, WHO/ NCD/NCS/99.2, World Health Organisation.

[8] Thomsen, T.F., Davidsen, M., Ibsen, H., Jorgensen, T., Jensen, G. and Borch-Johnsen, K. (2001) A new method for CHD prediction and prevention based on regional risk scores and randomized clinical trials; PRECARD and the Copenhagen Risk Score. Journal of Cardiovascular Risk, 8, 291-297. doi:10.1097/00043798-200110000-00008

[9] Toft, U., Kristoffersen, L., Lau, C., Borch-Johnsen, K. and Jørgensen, T. (2007) The dietary quality score: Validation and association with cardiovascular risk factors: The Inter99 study. European Journal of Clinical Nutrition, 61, 270-278. doi:10.1038/sj.ejen. 1602503

[10] Klatsky, A.L. (2010) Alcohol and cardiovascular health. Physiology \& Behavior, 100, 76-81. doi:10.1016/j.physbeh.2009.12.019

[11] Barefoot, J.C., Gronbaek, M., Feaganes, J.R., Mcpherson, R.S., Williams, R.B. and Siegler, I.C. (2002) Alcoholic beverage preference, diet, and health habits in the UNC Alumni Heart Study. American Journal of Clinical Nutrition, 76, 466-472.

[12] Johansen, D., Gronbaek, M., Overvad, K., Schnohr, P. and Andersen, P.K. (2005) Generalized additive models applied to analysis of the relation between amount and type of alcohol and all-cause mortality. European Journal of Epidemiology, 20, 29-36. doi:10.1007/s10654-004-2172-z

[13] Corrao, G., Rubbiati, L., Bagnardi, V., Zambon, A. and 
Poikolainen, K. (2000) Alcohol and coronary heart disease: A meta-analysis. Addiction, 95, 1505-1523. doi:10.1046/j.1360-0443.2000.951015056.x

[14] Kodama, S., Saito, K., Tanaka, S., et al. (2011) Alcohol consumption and risk of atrial fibrillation: A meta-analysis. Journal of the American College of Cardiology, 57, 427-436. doi:10.1016/j.jacc.2010.08.641

[15] Ronksley, P.E., Brien, S.E., Turner, B.J., Mukamal, K.J. and Ghali, W.A. (2011) Association of alcohol consumption with selected cardiovascular disease outcomes: A systematic review and meta-analysis. British Medical Journal, 342, d671. doi:10.1136/bmj.d671

[16] Feinman, L. and Lieber, C.S. (1999) Ethanol and lipid metabolism. American Journal of Clinical Nutrition, 70, 791792.

[17] Pennington, J.S., Shuvaeva, T.I. and Pennington, S.N. (2002) Maternal dietary ethanol consumption is associated with hypertriglyceridemia in adult rat offspring. Alcoholism: Clinical and Experimental Research, 26, 848-855. doi:10.1111/j.1530-0277.2002.tb02614.x

[18] Bessembinders, K., Wielders, J. and Van de Wiel, A. (2011) Severe hypertriglyceridemia influenced by alcohol (SHIBA). Alcohol and Alcoholism, 46, 113-116. doi:10.1093/alcalc/agq088

[19] Schroder, H., Ferrandez, O., Jimenez, C.J., Sanchez-Font, A. and Marrugat, J. (2005) Cardiovascular risk profile and type of alcohol beverage consumption: A population- based study. Annals of Nutrition and Metabolism, 49, 100-106. doi:10.1159/000084889

[20] Langer, R.D., Criqui, M.H. and Reed, D.M. (1992) Lipoproteins and blood pressure as biological pathways for effect of moderate alcohol consumption on coronary heart disease. Circulation, 85, 910-915. doi:10.1161/01.CIR.85.3.910

[21] Rimm, E.B., Williams, P., Fosher, K., Criqui, M. and Stampfer, M.J. (1999) Moderate alcohol intake and lower risk of coronary heart disease: Meta-analysis of effects on lipids and haemostatic factors. British Medical Journal, 319, 1523-1528. doi:10.1136/bmj.319.7224.1523

[22] Klatsky, A.L., Friedman, G.D. and Armstrong, M.A. (1986) The relationships between alcoholic beverage use and other traits to blood pressure: A new Kaiser Permanente study. Circulation, 73, 628-636. doi:10.1161/01.CIR.73.4.628

[23] Marques-Vida, P., Montaye, M., Haas, B., et al. (2001) Relationships between alcoholic beverages and cardiovascular risk factor levels in middle-aged men, the PRIME study. Atherosclerosis, 157, 431-440. doi:10.1016/S0021-9150(00)00734-6

[24] Stranges, S., Wu, T., Dorn, J.M., et al. (2004) Relationship of alcohol drinking pattern to risk of hypertension: A population-based study. Hypertension, 44, 813-819. doi:10.1161/01.HYP.0000146537.03103.f2 